Ciência eNatura, Santa Maria, v. 37 n. 3 set-dez. 2015, p. 877-888

Revista do Centro de Ciências Naturais e Exatas - UFSM

ISSNimpressa: 0100-8307 ISSN on-line: 2179-460X

\title{
Controle Ótimo de Sistemas Não Lineares com Aplicação no Cultivo do Milho.
}

\author{
Optimal Control of Nonlinear Systems with Application in Corn Cultivation.
}

\author{
Jéssica Camila Saldivia Bueno, Alexandre Molter, Luciana Rossato Piovesan \\ Universidade Federal de Pelotas - UFPel - Pelotas, RS
}

\begin{abstract}
Resumo
Este trabalho propõe umamodelagem matemática de sistemasdinâmicos biológicos controlados. São considerados dois modelosde interaçãoentre presa-predador, o de Lotka-Volterra e ode Holling-Tanner. A estratégia de controle utilizada é a de controle biológico e a aplicação se dá no cultivo do milho. O objetivo desta estratégia de controle é manter a população de pragas abaixo de nível de danos econômicos. O problema do controle é resolvido de duas maneiras, sendo que a primeiraconsidera o controle ótimo linear para um modelo generalizado aplicado ao caso dos sistemas linearizados e os não linearizados de Lotka-Volterra e Holling-Tanner e, a segundaconsiderauma estratégia de controle ótimo não linear aplicado no sistema de Lotka-Volterra.A contribuição deste trabalho para pesquisas em controle biológico de pragas é a modelagem matemática de problemas de controle ótimo linear e não linear e sua aplicação no cultivo do milho. A eficácia das estratégias de controle para os sistemas utilizados é verificada por meio de simulações numéricas.
\end{abstract}

Palavras-chave: Controle ÓtimoLinear, Controle Ótimo Não Linear, Modelo de Lotka-Volterra, Modelo de Holling-Tanner.

\begin{abstract}
This paper proposes a mathematical modelling of controlled biological dynamic systems. Two models of interaction between predator-prey are considered, the Lotka-Volterra and the Holling-Tanner. The control strategy used is biological control and the application occurs in corn cultivation. The purpose of this control strategy is to keep the pest population below economic injury level. The control problem is solved in two ways where the first considers the linear optimal control for a general model applied to the case of linearized and non-linearized systems of Lotka-Volterra and Holling-Tanner, and the second considers a strategy of nonlinear optimal control applied in the nonlinear Lotka-Volterra system. The contribution of thiswork toresearch inbiological pest controlis themathematical modeling oflinear and nonlinear optimal control problemsand itsapplicationin the cultivation ofcorn. The effectiveness of the control strategies for the systems used is verified by numerical simulations.
\end{abstract}

Keywords: Linear OptimalControl, Nonlinear Optimal Control, Lotka-VolterraModel, Holling-TannerModel. 


\section{Introdução}

$\mathrm{A}$

modelagem matemática de sistemas presa-predador é uma importante ferramenta nos estudos de problemas biológicos. Com o objetivo de descrever o comportamento interespecífico, surgiram diversos modelos matemáticos que retratam um sistema presa-predador. Estesmodelos presapredador têm sido amplamente empregados na modelagem deproblemas com controle biológico.

O controle biológico consiste em inserir predadores em meio às pragas, prejudiciais as plantações, com objetivo de manter a população de pragas abaixo do nível de danos econômicosbemcomo evitar a extinção das pragas, já que isso poderia influenciar no equilíbrio ecológico. De acordo com Bassanezi (2002) este controle visa minimizar ou evitar perdas econômicas.

Neste trabalho o controle biológico foi aplicadoem uma espécie de praga na lavoura de milho. Nessa aplicação, a presa é a lagarta do cartucho do milho, Spodoptera frugiperda, (Pereira, 2007) principal praga da lavoura de milho, e o predador, seu inimigo natural, o predadorTrichogramma. A vespaTrichogramma é um predador natural da lagarta do cartucho do milho (Cruz \& Monteiro, 2004). A vespa Trichogramma parasita os ovos das mariposas (fase adulta da lagarta do cartucho do milho) e, com isso, impede o nascimento de novas lagartas. O ciclo de vida davespa é em torno de 10 dias. Já o ciclo de vida da presa (lagarta do cartucho do milho) é em torno de 30 dias e está descrito na Tabela 1.

Tabela 1: Ciclo Biológico Spodoptera frugiperda

\begin{tabular}{c|c}
\hline \multicolumn{2}{c}{ Ciclo biológico Spodoptera frugiperda } \\
\hline $\begin{array}{c}\text { Incubação } \\
\text { dos ovos }\end{array}$ & 3 dias \\
\hline Período larval & 15 dias \\
\hline $\begin{array}{c}\text { Média de ovos } \\
\text { por fêmea }\end{array}$ & 100 ovos \\
\hline Ciclo total & 30 dias \\
\hline
\end{tabular}

Fonte: Comunicado Técnico 114, EMBRAPA. (Cruz \& Monteiro, 2004)
SegundoTang e Cheke(2008), a aplicação do controle biológico na lavoura se dá logo após aparecerem os primeiros focos de pragas evitando, assim, picos de infestação que seriam prejudiciais à cultura.No caso do cultivo do milho, ao aparecerem as primeiras mariposas é recomendado iniciar a aplicação do controle biológico. Na bibliografia de manejo de pragas do milho não foi encontrada qual o nível de pragas aceitável na cultura de milho. No entanto, após a leitura de diversos autores fez-se um estimativa para o nível de pragas que não causem danos econômicos a lavoura. Estimou-se que este nível é de,em média, sete lagartas do cartucho do milho por metro quadrado, ou seja, valores superiores a esta densidade causam danos econômicos à lavoura.

Diversos trabalhos tratam do controle de pragas por meios biológicos, dentre eles, destacase o de o de Tang e Cheke (2008), onde o controle de pragas foi feito de forma integrada, por meios biológicos e com inseticidas, e os controladores são não lineares; o de Cardoso et.al (2009) que propôs o controle biológico de pragas da soja utilizando otimização evolucionária multiobjetivo; o de Molter e Rafikov (2011) e Molter e Rafikov (2014) que propuseram o controle biológico de pragas em lavouras de soja utilizando controle ótimo não linear. Já o controle biológico de pragas em lavouras de milho foi tratado em Cruz e Monteiro (2004) e Pereira (2007). No entanto, nestes trabalhos não foi utilizada a modelagem matemática do problema de controle. No presente trabalho busca-se o controle biológico da praga do milho descrevendo o problema através de modelos matemáticos. Além disto, a abordagem do controle ótimo linear e não linear nesta aplicação é um diferencial deste trabalho.

O objetivo deste trabalho é formular e resolver dois problemas de controle ótimo para sistemas presa-predador com aplicação no controle biológico de pragas. $\mathrm{O}$ primeiro problema visa utilizar controle ótimo linear paraum modelo generalizado em sistemas linearizados e não linearizados de Lotka-Volterra e Holling-Tanner. $\mathrm{O}$ segundo utiliza controle ótimo não linear aplicado no sistema de LotkaVolterra.

Por meio de simulações numéricas se buscará manter os sistemas em níveis de pragas 
desejados para o equilíbrio biológico e abaixo de danos econômicos.

\section{Modelo Generalizadode Interação entre Espécies}

No propósitode descrever a dinâmica ecológica, entre duas ou mais espécies, surgiram diversos modelos de interação interespecífica, tendo como um dos precursores o modelo presapredador de Lotka-Volterra formulado por Lotka em 1925 (Lotka, 1925) e Volterra em 1927 (Volterra, 1927). Posteriormente, diversos modelos foram criados na tentativa de generalizálo. Em 1936, surge o modelo geral de Kolmogorov (Bassanezi, 2002) que pode ser descrito da seguinte forma:

$$
\dot{x}_{i}=x_{i} f_{i}\left(x_{1}, \ldots, x_{i}, \ldots, x_{n}\right) \text { para } i=1,2, \ldots, n,
$$

onde $x_{i}$ representa a densidade populacional de determinada espéciei e as funções $f_{i}$ representam as relaçõesinterespecíficasde cada espécie.

A partir, do modelo geral de Kolmogorov, surgiram modelos particulares que se diferenciam pela relação interespecífica, isto é, pela função $f_{i}$ (Bassanezi, 2002). Entre eles podemos destacar o modelo de Holling-Tanner formulado por Holling em 1959 (Holling, 1959) e adaptado por Tanner em 1975 (Tanner, 1975). Sendo este, bem como o modelo de LotkaVolterra casos particulares do modelo de Kolmogorov.

Neste trabalho, estamos utilizando dois modelos presa-predador distintos, Lotka-Volterra e Holling-Tanner, ambos provém do modelo geral de Kolmogorov, que para este caso tem a seguinte forma:

$$
\begin{aligned}
& \dot{x}_{1}=x_{1} f\left(x_{1}, x_{2}\right), \\
& \dot{x}_{2}=x_{2} g\left(x_{1}, x_{2}\right),
\end{aligned}
$$

onde $x_{1}$ e $x_{2}$ representam, respectivamente, a densidade de presas e predadores.

\subsection{Modelo de Lotka-Volterra}

O modelo de Lotka-Volterra presa-predador pode ser descrito da seguinte forma:

$$
\begin{aligned}
& \dot{x}_{1}=x_{1}\left(r_{1}-a_{11} x_{1}-a_{12} x_{2}\right), \\
& \dot{x}_{2}=x_{2}\left(-r_{2}+a_{21} x_{1}+a_{22} x_{2}\right)
\end{aligned}
$$

onde $x_{1}$ e $x_{2}$ são respectivamente as populações de presa (lagarta do cartucho do milho) dado pelo número de lagartas por metro quadrado, e seu predador (vespaTrichogramma- inimigo natural) dado pelo número de vespas por metro quadrado.

$O$ coeficiente $r_{1}$ caracteriza a taxa de reprodução da lagarta do cartucho de milho (presa); $r_{2}$ caracteriza a taxa de mortalidade natural de vespas (predador). Os coeficientes $a_{i j}$, com $i, j=1,2$, caracterizam as taxas de interações entre as espécies do sistema, sendo que nesta situação o $a_{22}=0$, pois os predadores não competem entre si.Os valores dos coeficientes são calculados com base nos dados apresentados na Tabela 1.Segundo o modelo de Malthus, temos $\frac{d N}{d t}=r N$, onde $r$ é a taxa de crescimento ou decrescimento de uma determinada população $N$, dependendo se é positivo ou negativo respectivamente (Boyce \& Diprima, 2002).

Uma solução para o modelo de Malthus é $N=$ $N_{0} e^{r_{1} t}$, onde $N_{0}$ é a condição inicial. A partir dos dados da Tabela 1 , temos $100=1 \cdot e^{30 r_{1}}$, isto é, $\ln (100)=30 r_{1}$, obtendo-se $r_{1}=\frac{\ln (100)}{30}=0,15$. A expectativa de vida davespaTrichogramma é em torno de 10 dias, portando, o coeficiente de mortalidade é $r_{2}=\frac{1}{10}=0,1$.Segundo Tusset e Rafikov (2004), os valores de $a_{11}, a_{12}$ e $a_{21}$ podem ser calculados com base nas taxas de crescimento e parâmetros de interação de cada espécie. Considerando $a_{11}=\frac{r_{1}}{K_{1}}$, onde $K_{1}=160$ é a capacidade suporte de presas, obtemos $a_{11}=$ 0,0009. Seja $a_{12}=a_{11} \alpha$, onde $\alpha=40$ é o parâmetro de interação entre presas e predadores, obtemos $a_{12}=0,036$. Para $a_{21}=\frac{r_{2}}{K_{2}}$, onde $K_{2}=65$ é capacidade suporte de predadores, temos $a_{21}=$ 0,0015 .Os parâmetros $K_{1}, K_{2}$ e $\alpha$ foram obtidos de Tusset,et al.(2004), cuja fonte é bibliográfica.

A obtenção de parâmetros por meios experimentais, de fato, não é simples.O parâmetro $r_{1}$ é estimado para uma população em crescimento exponencial ideal, onde todos os ovos são passíveis de gerarem indivíduos que chegam a fase adulta. Do ponto de vista biológico, este seria o pior caso para uma infestação de pragas, visto que a geração de novos indivíduos estaria livre de perdas. Em cima desta estimativa para $_{1}$, determinada a partir dos dados da Tabela 1 , outros parâmetros podem ser calculados. Já os valores das capacidades de suporte $K_{1}$ e $K_{2}$, devem ser obtidos de forma experimental. Neste 
trabalho fez-se uma estimativa destes a partir da leitura de trabalhos que tratam sobre o cultivo do milho.

As taxas de interações entre as espécies do sistema $\left(a_{i j}\right)$ são calculados a partir de outros parâmetros inerentes a estas, que são a capacidade de suporte de cada espécie $\left(K_{1}\right.$ e $\left.K_{2}\right)$ e parâmetros de interação $(\alpha)$, estes que também devem advir de experimentos reais, mas neste trabalho foram obtidos da literatura.

Os valores dos coeficientes do modelo (2) estão organizados na Tabela 2.

Tabela 2: Valores dos Coeficientes para o Modelo de Lotka-Volterra

\begin{tabular}{c|c|c|c|c}
\hline $\boldsymbol{r}_{\mathbf{1}}$ & $\boldsymbol{r}_{\mathbf{2}}$ & $\boldsymbol{a}_{\mathbf{1 1}}$ & $\boldsymbol{a}_{\mathbf{1 2}}$ & $\boldsymbol{a}_{\mathbf{2 1}}$ \\
\hline 0,15 & 0,1 & 0,0009 & 0,036 & 0,0015 \\
\hline
\end{tabular}

Atribuindo os valores dos coeficientes da Tabela 2ao sistema (2) obtém-se:

$$
\begin{aligned}
& \dot{x}_{1}=x_{1}\left(0,15-0,0009 x_{1}-0,036 x_{2}\right), \\
& \dot{x}_{2}=x_{2}\left(-0,1+0,0015 x_{1}\right) .
\end{aligned}
$$

O sistema (3) é quase linear na vizinhança de cada ponto crítico. Para analisar a estabilidade do sistema (3), primeiramente determinamos os pontos críticos, de onde encontramos três: $P_{1}=$ $(0,0), P_{2}=(8,0)$ e $P_{3}=(66,67 ; 2,5)$.

Analisando a estabilidade dos pontos $P_{1}$ e $P_{2}$ verificou-se que são instáveis. Também se observou que o ponto $P_{1}$ não tem significado biológico, no viés de controle biológico de pragas, uma vez que não existem presas e predadores.

Em $P_{2}$ temos a ausência de predadores, tornando-se assim, inviável a aplicação do controle biológico.Em contra partida em $P_{3}$ há tanto presas quanto predadores, sendo este o único ponto de interesse para análise.

Aplicando Série de Taylor no sistema (3) em torno de $P_{3}=(66,67 ; 2,5)$, obtemos, o seguinte sistema linear:

$$
\begin{aligned}
& \dot{y}_{1}=-0,06 y_{1}-2,4 y_{2}, \\
& \dot{y}_{2}=0,00375 y_{1}-5 \times 10^{-6} y_{2},
\end{aligned}
$$

de onde obtém-se que $y_{1}=x_{1}-66,67$ e $y_{2}=x_{2}-$ 2,5 .

Os autovalores do sistema linear (3) são complexos com parte real negativa. Portanto, temos um ponto espiral assintoticamente estável em $P_{3}=(66,67 ; 2,5)$, deste modo o sistema (3) irá estabilizar-se em $P_{3}=(66,67 ; 2,5)$, entretanto 66,67 é um número elevado de pragas para a lavoura de milho, isto é, com esse número de lagartas do cartucho, a lavoura terá sérios danos. Neste caso, será necessário aplicar uma estratégia de controle a fim de diminuir o número de lagartas do cartucho obtendo, assim, um ponto de equilíbrio desejável que não cause danos à plantação de milho.

Na figura 1 é apresentada a dinâmica do sistema de Lotka-Volterra sem controle (3), com condições iniciais em $(2,2)$, que se estabiliza em um nível superior ao recomendado, ocasionando danos à lavoura.

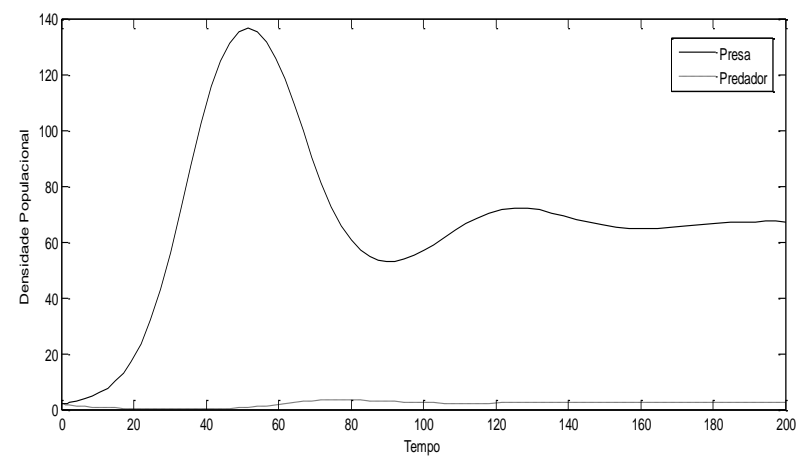

Figura 1: Trajetórias do Sistema de LotkaVolterra sem Controle.

\subsection{Modelode Holling-Tanner}

O modelo de Holling-Tanner para uma presa e um predador pode ser descrito como:

$$
\begin{aligned}
& \dot{x}_{1}=x_{1}\left(a-\frac{a x_{1}}{K}-\frac{b x_{2}}{\left(D+x_{1}\right)}\right) \\
& \dot{x}_{2}=x_{2}\left(\frac{\beta x_{1}}{\left(D+x_{1}\right)}-\gamma\right),
\end{aligned}
$$

Os coeficientes $a, K, b, \beta, D, \gamma$ representam a taxa intrínseca de crescimento de presas, capacidade suporte da população de presas, taxa de captura, taxa máxima do crescimento de predadores, constante da metade da saturaçãode presas e taxa de mortalidade do predador, respectivamente.A taxa de captura $b$ pode ser obtida da primeira equação do sistema (4) em um ponto de equilíbrio desejado $\left(x_{1}^{*}, x_{2}^{*}\right)$. Daí, temos:

$$
b=\left(a-\frac{a}{k} x_{1}^{*}\right)\left(x_{1}^{*}+D\right) \frac{1}{x_{2}^{*}} \text {. }
$$


Considerando os valores de $a, K, D$ daTabela 3e o ponto de equilíbrio desejado $(6,7)$, a escolha deste ponto será justificada na próxima seção, obtemos $b=0,65$. Os demais coeficientes podem ser obtidos em Tusset, et al. (2004).

Tabela 3: valores dos coeficientes para o modelo de Holling-Tanner.

\begin{tabular}{c|c|c|c|c|c}
\hline $\boldsymbol{a}$ & $\boldsymbol{K}$ & $\boldsymbol{b}$ & $\boldsymbol{\beta}$ & $\boldsymbol{D}$ & $\boldsymbol{\gamma}$ \\
\hline 0,15 & 10 & 0,65 & 0,004 & 70 & 0,003 \\
\hline
\end{tabular}

Atribuindo os valores dos coeficientes da Tabela 3 ao sistema (4) obtém-se:

$$
\begin{aligned}
& \dot{x}_{1}=x_{1}\left(0,15-0,015 x_{1}-\frac{0,65 x_{2}}{\left(70+x_{1}\right)}\right), \\
& \dot{x_{2}}=x_{2}\left(\frac{0,004 x_{1}}{\left(70+x_{1}\right)}-0,003\right),
\end{aligned}
$$

Para análise da estabilidade do sistema (5) encontramos três pontos críticos: $P_{1}=(0,0), P_{2}=$ $(10,0)$ e $P_{3}=(210,-1292)$. Observa-se que $P_{3}$ não é um ponto de equilíbrio biologicamente viável. A partir da análise da estabilidade o ponto $P_{1}$ é instável e o ponto $P_{2}$ é estável. O equilíbrio natural do sistema (5), como pode ser visto na figura 2, é o ponto $P_{2}=(10,0)$. Pode-se observar que este ponto não é desejado por manter o nível de presas acima do aceitável para danos econômicos, o que justifica aplicação do controle no sistema. Além disto, este ponto mostra que os predadores são levados à extinção, o que também não é desejável ao equilíbrio biológico das espécies.

Para os parâmetros considerados acima e condições iniciais em $(2,2)$ obtemos os gráficos das populações de presa e predador, conforme Figura 2.

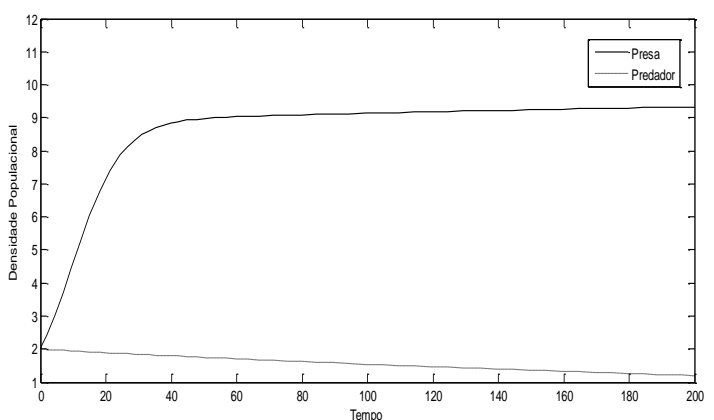

Figura 2:Trajetórias do Sistema de HollingTanner sem Controle.
Durante as simulações foram feitos alguns testes com o sistema (5) para verificar a relação entre os coeficientes $K$ e $D$. No sistema (4), considerando o estado de equilíbrio, atribuindo os valores dos coeficientes e o equilíbrio desejado, e o valor de $K$ ou de $D$, podemos calcular o outro(ou $K$, ou $D$ ). Desta forma, observou-se que aumentando ou diminuindo os valores de Dtambém aumenta ou diminui $K$, respectivamente. Então para um determinado $K$ devemos ter um $D$ específico correspondente.

\section{Controle Ótimo Linear Aplicado à Sistemas Não Lineares}

Como vimos nas subseções 2.1 e 2.2 os modelos de Lotka-Volterra (3) e Holling-Tanner (5) estabilizam-se em um nível de pragas superior ao recomendado, sendo necessário a aplicação de controle.

Consideramos $\left(x_{1}^{*}, x_{2}^{*}\right)$ o ponto de equilíbrio desejado. Escolhemos $x_{1}^{*}=6$, sendo este valor abaixo do nível de danos econômicos. O valor de $x_{2}^{*}$ pode ser encontrado na primeira equação do modelo a ser considerado. Para o modelo de Lotka-Volterra (2) temos $\quad x_{2}^{*}=\frac{r_{1}-a_{11} x_{1}^{*}}{a_{12}}$. Considerando os parâmetros da tabela obtemos $x_{2}^{*}=4$, logo o estado de equilíbrio desejado para os sistema de Lotka-Volterra (3) é $\left(x_{1}^{*}, x_{2}^{*}\right)=(6,4)$. Já para o modelo de Holling-Tanner (4) temos $x_{2}^{*}=-\frac{a\left(D+x_{1}^{*}\right)\left(x_{1}^{*}-K\right)}{b K}$, para o qual, com base na Tabela 3, encontramos $x_{2}^{*}=7$. Então, o estado de equilíbrio desejado para o sistema de HollingTanner (5) é dado por $\left(x_{1}^{*}, x_{2}^{*}\right)=(6,7)$.

\subsection{Controle Ótimo Linear para osSistemasLinearizados}

O sistema de Kolmogorov (1) com controle é descrito da seguinte forma(Tusset \&Rafikov, 2004):

$$
\begin{aligned}
& \dot{x}_{1}=x_{1} f\left(x_{1}, x_{2}\right), \\
& \dot{x}_{2}=x_{2} g\left(x_{1}, x_{2}\right)+U,
\end{aligned}
$$

onde $U=u+u^{*}$.

Seja $\left(x_{1}^{*}, x_{2}^{*}\right)$ o estado de equilíbrio desejado para sistema (1) tal que : 


$$
\begin{aligned}
& f\left(x_{1}^{*}, x_{2}^{*}\right)=0, \\
& x_{2}^{*} f\left(x_{1}^{*}, x_{2}^{*}\right)+u^{*}=0,
\end{aligned}
$$

onde,

$$
u^{*}=-x_{2}^{*} g\left(x_{1}^{*}, x_{2}^{*}\right)
$$

Então, o problema do controle ótimo está em encontrar a função de controle $u$ que transfere o sistema (6) do estado inicial (Naidu, 2003):

ao estado final:

$$
Y(0)=Y_{0}
$$

$$
Y(\infty)=0,
$$

e minimiza o funcional:

$$
J=\int_{0}^{\infty}\left[Y^{T} Q Y+u R u\right] d t
$$

onde $Y=\left[\begin{array}{l}y_{1} \\ y_{2}\end{array}\right]=\left[\begin{array}{l}x_{1}-x_{1}^{*} \\ x_{2}-x_{2}^{*}\end{array}\right], \quad R=[1] \quad$ e $\quad Q=$ $\left[\begin{array}{cc}q_{11} & 0 \\ 0 & q_{22}\end{array}\right]$, sendo $Q$ uma matriz semi-definida positiva. Em geral as matrizes $Q$ e $R$ são denominadas de matrizes de peso, ou de ponderação (Naidu, 2003). Nesta aplicação elas estão relacionadas ao custo social das populações e o custo do controle a ser aplicado, respectivamente (Caetano \&Yoneyama, 2001; Rodrigues, Monteiro \& Torres, 2010).

Aplicando o controle deseja-se que o sistema estabilize no nível de pragas desejado à medida que a perturbação $x-x^{*}$ tenda a zero.

Linearizando o sistema (6) em torno do ponto de equilíbrio desejado $\left(x_{1}^{*}, x_{2}^{*}\right)$, temos:

$$
\begin{aligned}
& \dot{x}_{1}=x_{1}^{*} f\left(x_{1}^{*}, x_{2}^{*}\right)+f\left(x_{1}^{*}, x_{2}^{*}\right)\left(x_{1}-x_{1}^{*}\right) \\
& +x_{1}^{*} f_{x_{1}}^{\prime}\left(x_{1}^{*}, x_{2}^{*}\right)\left(x_{1}-x_{1}^{*}\right)+x_{1}^{*} f_{x_{2}}^{\prime}\left(x_{1}^{*}, x_{2}^{*}\right)\left(x_{2}-x_{2}^{*}\right), \\
& \dot{x}_{2}=x_{2}^{*} g\left(x_{1}^{*}, x_{2}^{*}\right)+g\left(x_{1}^{*}, x_{2}^{*}\right)\left(x_{2}-x_{2}^{*}\right) \\
& +x_{2}^{*} g_{x_{1}}^{\prime}\left(x_{1}^{*}, x_{2}^{*}\right)\left(x_{1}-x_{1}^{*}\right)+x_{2}^{*} g_{x_{2}}^{\prime}\left(x_{1}^{*}, x_{2}^{*}\right)\left(x_{2}-x_{2}^{*}\right) \\
& -x_{2}^{*} g\left(x_{1}^{*}, x_{2}^{*}\right)+u .
\end{aligned}
$$

Reescrevendo o sistema linearizado (7) em forma matricial tem-se:

onde

$$
\dot{Y}=A Y+B u,
$$

$$
A=\left[\begin{array}{cc}
x_{1}^{*} f_{x_{1}}^{\prime}\left(x_{1}^{*}, x_{2}^{*}\right) & x_{1}^{*} f_{x_{2}}^{\prime}\left(x_{1}^{*}, x_{2}^{*}\right) \\
x_{2}^{*} g_{x_{1}}^{\prime}\left(x_{1}^{*}, x_{2}^{*}\right) & x_{2}^{*} g_{x_{2}}^{\prime}\left(x_{1}^{*}, x_{2}^{*}\right)+g\left(x_{1}^{*}, x_{2}^{*}\right)
\end{array}\right]
$$

$\mathrm{eB}=\left[\begin{array}{ll}0 & 1\end{array}\right]^{T}$.

Neste caso a função de controle $u$ pode ser obtida da seguinte lei de controle ótimo:

$$
u=-R^{-1} B^{T} S Y,
$$

onde a matriz $S$, simétrica, definida positiva, é a solução da equação algébrica matricial de Ricatti:

$$
S A+A^{T} S-S B R^{-1} B^{T} S+Q=0 .
$$

\subsubsection{Sistema Linearizado de Lotka- Volterra}

Considerando o sistema de Lotka-Volterra com controle (6) e escrevendo na forma matricial (8), obtém-se:

$$
\left[\begin{array}{l}
\dot{y}_{1} \\
\dot{y}_{2}
\end{array}\right]=\left[\begin{array}{cc}
0,0009 x_{1}^{*} & -0,036 x_{1}^{*} \\
0,0015 x_{2}^{*} & -0,1+0,0015 x_{1}^{*}
\end{array}\right]\left[\begin{array}{l}
y_{1} \\
y_{2}
\end{array}\right]+B u,
$$

ondeB $=\left[\begin{array}{ll}0 & 1\end{array}\right]^{T}$ e no ponto de equilíbrio desejável $(6,4)$, temos:

$$
A=\left[\begin{array}{cc}
-0,0054 & -0,216 \\
0,006 & -0,091
\end{array}\right]
$$

escolhendo:

$$
Q=\left[\begin{array}{cc}
10 & 0 \\
0 & 10
\end{array}\right] \mathrm{e} R=[1]
$$

Calculando a matrizSatravés da equação de Riccati (9), obtém-se:

$$
S=\left[\begin{array}{cc}
48,0695 & -3,0731 \\
-3,0731 & 3,2759
\end{array}\right]
$$

A função de controle ótimou do sistema tem a seguinte forma:

$$
u=3,0731 y_{1}-3,2749 y_{2} \text {. }
$$

Em termos de aplicação, o resultado obtido para $u$ representa a quantidade de predadores por metro quadrado que devem ser introduzidos na lavoura por dia.

As simulações numéricas para todos os casos apresentados neste trabalho foram realizadas no software Matlab, utilizando a função $L Q R$ para resolver a equação de Riccati e Runge-Kutta de quarta ordem para a resolução do sistema.

As trajetórias temporais do sistema com controle (10) são apresentadas na Figura 3, com condições iniciais em $(2,2)$. Observa-se que o sistema controlado aproxima-se do seu estado de equilíbrio em torno de 15 dias. 


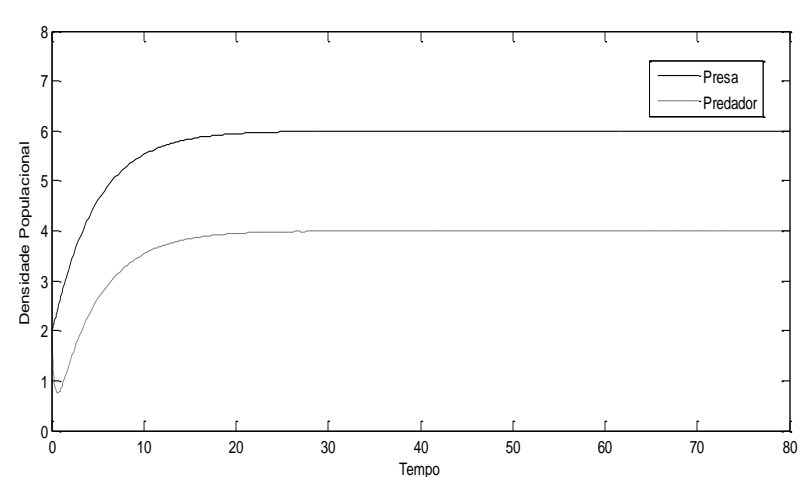

Figura 3:Trajetórias do Sistema Linearizado de Lotka-Volterra com Controle.

\subsubsection{Sistema Linearizado de Holling- Tanner}

Considerando o sistema (4) em um estado de equilíbrio desejado $\left(x_{1}^{*}, x_{2}^{*}\right)$, temos:

$$
\begin{aligned}
& x_{1}^{*}\left(a-\frac{a x_{1}^{*}}{K}-\frac{b x_{2}^{*}}{\left(D+x_{1}^{*}\right)}\right)=0, \\
& x_{2}^{*}\left(\frac{\beta x_{1}^{*}}{\left(D+x_{1}^{*}\right)}-\gamma\right)+u^{*}=0,
\end{aligned}
$$

onde $u^{*}=-x_{2}^{*}\left(\frac{\beta x_{1}^{*}}{\left(D+x_{1}^{*}\right)}-\gamma\right)$.

Escrevendo osistema de Holling-Tanner linearizado (4) em forma matricial (8), obtém-se:

$$
\left[\begin{array}{l}
\dot{y}_{1} \\
\dot{y}_{2}
\end{array}\right]=\left[\begin{array}{cc}
\left(-\frac{a}{K}+\frac{b x_{2}^{*}}{\left(D+x_{x}^{*}\right)^{2}}\right) x_{1}^{*} & -\frac{b x_{1}^{*}}{\left(D+x_{1}^{*}\right)} \\
\frac{D \beta x_{2}^{*}}{\left(D+x_{1}^{*}\right)^{2}} & \frac{\beta x_{1}^{*}}{\left(D+x_{1}^{*}\right)}-\gamma
\end{array}\right]\left[\begin{array}{l}
y_{1} \\
y_{2}
\end{array}\right]+B u,
$$

onde $B=\left[\begin{array}{ll}0 & 1\end{array}\right]^{T}$ e no ponto de equilíbrio desejável $(6,7)$, temos:

$$
A=\left[\begin{array}{cc}
-0,085 & -0,513 \\
0,00034 & -0,0027
\end{array}\right]
$$

escolhendo:

$$
Q=\left[\begin{array}{cc}
10 & 0 \\
0 & 10
\end{array}\right] \mathrm{e} R=[1]
$$

Calculando a matriz $S$ por meio da equação de Riccati(9), obtém-se:

$$
S=\left[\begin{array}{cc}
54,5432 & -08527 \\
-0,8527 & 3,1733
\end{array}\right] .
$$

A função de controle ótimo $u$ do sistema tem a seguinte forma:

$$
u=0,8527 y_{1}-3,1733 y_{2} .
$$

As trajetórias temporais do sistema linearizado de Holling-Tanner com controle (11) são apresentadas na Figura 4, com condições iniciais com condições iniciais em $(2,2)$. É possível notar que o sistema foi controlado e atinge o seu equilíbrio desejado em menos de 25 dias.

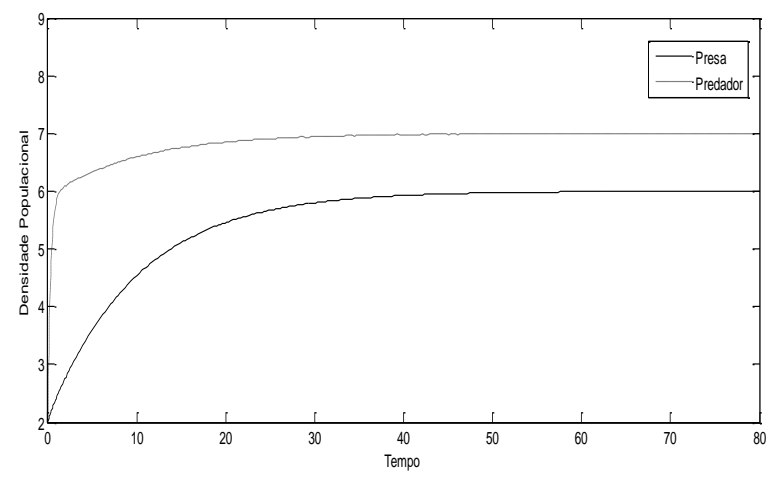

Figura 4: Trajetórias do Sistema Linearizado de Holling-Tanner com Controle.

\subsection{Controle Ótimo Linearpara SistemasNão Linearizados}

O sistema (1) com controle pode ser descrito da seguinte forma matricial(Rafikov, Balthazar \& Von Bremen, 2008):

$$
\dot{X}=A X+G(X)+B U,
$$

onde $A \in \mathbb{R}^{2 \times 2}$ é uma matriz constante, $G(X) \in \mathbb{R}^{2}$ é um vetor cujos elementos são funções não lineares contínuas, $B \in \mathbb{R}^{2} \mathrm{e} U \in \mathbb{R}^{2}$ tal que $U=u+u^{*}$.

Considerando $X^{*}=\left[x_{1}^{*} x_{2}^{*}\right]^{T}$ o estado de equilíbrio desejado, segue-se:

$$
A X^{*}+G\left(X^{*}\right)+B u^{*}=0 .
$$

O controle $u^{*}$ tem a seguinte forma:

$$
u^{*}=-A X^{*}-G\left(X^{*}\right) \text {. }
$$

Definimos

$$
Y=\left[\begin{array}{l}
y_{1} \\
y_{2}
\end{array}\right]=\left[\begin{array}{l}
x_{1}-x_{1}^{*} \\
x_{2}-x_{2}^{*}
\end{array}\right]
$$

ondeYé a perturbação no sistema (12) em torno do estado de equilíbrio desejado. Substituindo as novas variáveis (15) em (12) e considerando (13)(14), obtemos as seguintes equações:

$$
\dot{Y}=A Y+g(Y)+B u \text {. }
$$


Teorema1. Se existem matrizes $Q$ e $R$, definidas positivas, sendo $Q$ simétrica, tal que a função,

$$
L(Y)=Y^{T} Q Y-g^{T}(Y) S Y-Y^{T} S g(Y),
$$

é definida positiva. Então o controle linear feedback:

$$
u=-R^{-1} B^{T} S Y,
$$

é ótimo, no sentido de transferir o sistema não linear (12) de um estado inicial,

$$
Y(0)=Y_{0}
$$

a um estado final:

$$
Y(\infty)=0
$$

minimizando o funcional:

$$
J=\int_{0}^{\infty}\left[L(Y)+u^{T} R u\right] d t,
$$

onde a matrizS, simétrica, definida positiva,é a solução da equação algébrica matricial de Ricatti (9).

A prova do Teorema1 pode ser encontrada em Rafikov,et al. ( 2008).

\subsubsection{Controle do Sistema de Lotka-Volterra}

Reescrevendo o sistema de Lotka-Volterra (2) na forma matricial (16), obtemos:

$\mathrm{e}$

$$
A=\left[\begin{array}{cc}
-a_{11} x_{1}^{*} & -a_{12} x_{1}^{*} \\
-a_{21} x_{2}^{*} & -r_{2}+a_{21} x_{1}^{*}
\end{array}\right]
$$

ondeB $=\left[\begin{array}{ll}0 & 1\end{array}\right]^{T}$

$$
g(Y)=\left[\begin{array}{l}
-a_{11} y_{1}^{2}-a_{12} y_{1} y_{2} \\
-a_{21} y_{2} y_{1}-a_{22} y_{2}^{2}
\end{array}\right],
$$

Considerando os parâmetros da Tabela 2 e o ponto de equilíbrio desejado $(6,4)$ a matriz $A(17)$ tem a seguinte forma:

$$
A=\left[\begin{array}{cc}
-0,0054 & -0,216 \\
0,006 & -0,091
\end{array}\right] .
$$

Escolhendo,

$$
Q=\left[\begin{array}{cc}
10 & 0 \\
0 & 10
\end{array}\right] \mathrm{e} R=[1],
$$

obtemos:

$$
S=\left[\begin{array}{rr}
48,0695 & -3,0731 \\
-3,0731 & 3,2759
\end{array}\right] .
$$

Então, a função de controle tem a seguinte forma:

$$
u=3,0731 y_{1}-3,2759 y_{2} .
$$

As trajetórias temporais do sistema de LotkaVolterra com controle (12) são apresentadas na Figura 5. É possível notar que o sistema foi controlado e as trajetórias atingem o equilíbrio em aproximadamente 20 dias

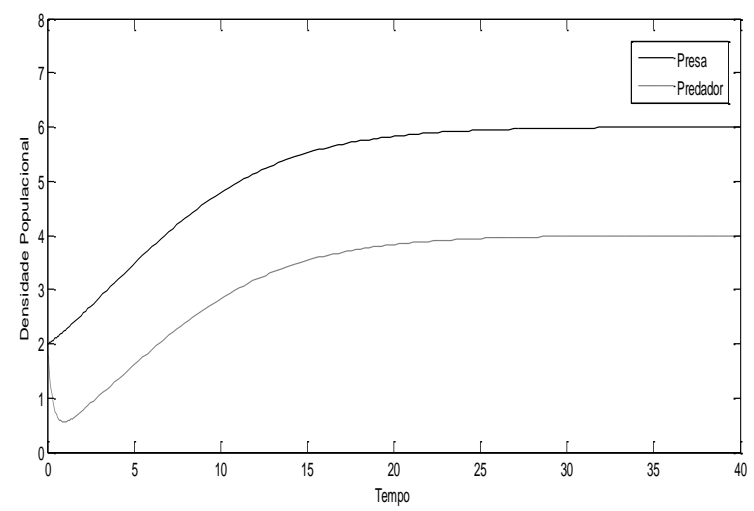

Figura 5: Trajetórias do Sistema não Linearizado de Lotka-Volterra com Controle.

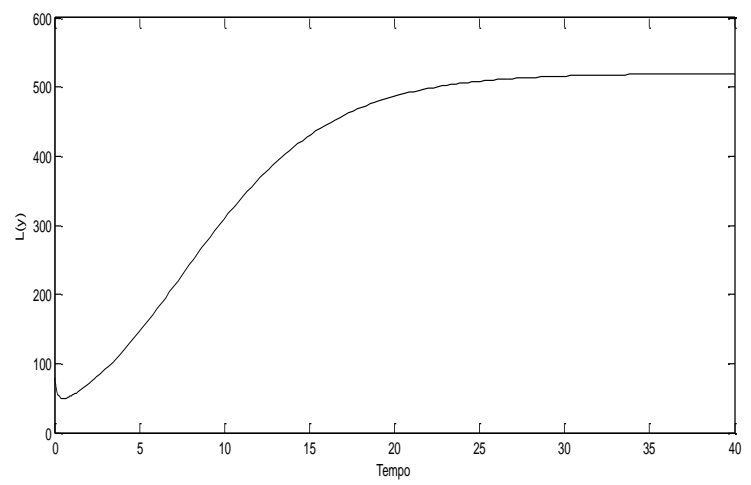

Figura 6: Função Definida Positiva $L(Y)$ para o Modelo de Lotka-Volterra.

\subsubsection{Controle do Sistema de Holling- Tanner}

Reescrevendo o sistema (4) como a equação (12), obtemos: 


$$
A=\left[\begin{array}{cc}
-\frac{a x_{1}^{*}}{K} & 0 \\
0 & -\gamma
\end{array}\right]
$$

e

$$
g(Y)=\left[\begin{array}{l}
g_{11} \\
g_{21}
\end{array}\right]
$$

onde

$$
\begin{gathered}
g_{11}=a y_{1}-\frac{a\left(y_{1}+x_{1}^{*}\right)^{2}-a x_{1}^{*} x_{1}^{*}-a x_{1}^{*} y_{1}}{k} \\
-\frac{b\left(y_{1}+x_{1}^{*}\right)\left(y_{2}+x_{2}^{*}\right)}{\left(D+y_{1}+x_{1}^{*}\right)}+\frac{b x_{1}^{*} x_{2}^{*}}{\left(D+x_{1}^{*}\right)^{\prime}} \\
g_{21}=\frac{\beta\left(y_{1}+x_{1}^{*}\right)\left(y_{2}+x_{2}^{*}\right)}{\left(D+y_{1}+x_{1}^{*}\right)}-\frac{\beta x_{1}^{*} x_{1}^{*}}{\left(D+x_{1}^{*}\right)^{\prime}}
\end{gathered}
$$

$\mathrm{eB}=\left[\begin{array}{ll}0 & 1\end{array}\right]^{T}$

Considerando os parâmetros da Tabela 3 e o ponto de equilíbrio desejado $(6,4)$ a matriz $A$ (18) tem a seguinte forma:

$$
A=\left[\begin{array}{cc}
-0,09 & 0 \\
0 & -0,003
\end{array}\right]
$$

Escolhendo $Q=\left[\begin{array}{cc}10 & 0 \\ 0 & 10\end{array}\right] \mathrm{e} R=[1]$. Obtemos:

$$
S=\left[\begin{array}{cc}
55,56 & 0 \\
0 & 3,1593
\end{array}\right]
$$
forma:

Então, a função de controle tem a seguinte

$$
u=3,1593 y_{2} .
$$

As trajetórias temporais do sistema HollingTanner com controle (12) são apresentadas na Figura7 e é possível notar que o sistema foi controlado e atinge o seu equilíbrio desejado em torno de 40 dias.

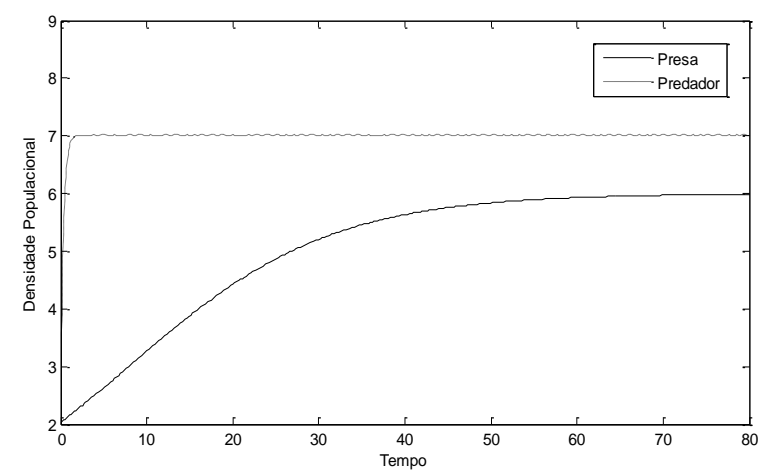

Figura 7: Trajetórias do Sistema não Linearizado de Holling-Tanner com Controle.

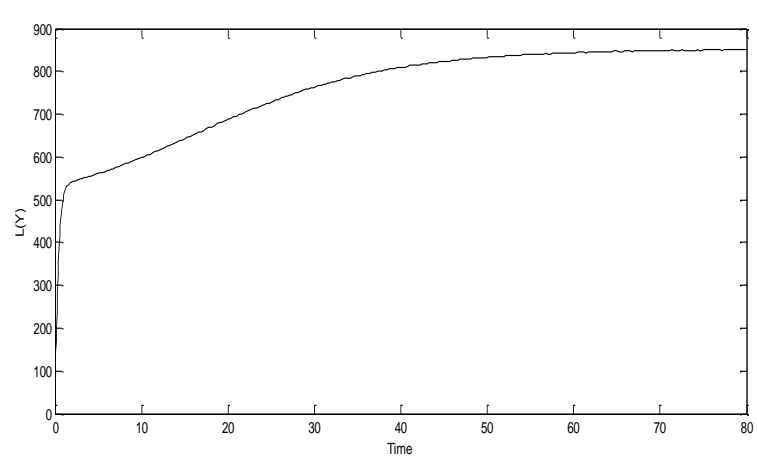

Figura 8: Função Definida Positiva $L(Y)$ para o Modelo de Holling-Tanner.

\section{Controle Ótimo Não Linear}

O modelo de Lotka-Volterra com controle ótimo não linear pode ser descrito da seguinte forma:

$$
\begin{aligned}
& \dot{x}_{1}=x_{1}\left(r_{1}-a_{11} x_{1}-a_{12} x_{2}\right) \\
& \dot{x}_{2}=x_{2}\left(r_{2}-a_{21} x_{1}-a_{22} x_{2}+u+u^{*}\right)
\end{aligned}
$$

onde $x_{1}$ e $x_{2}$ representam, respectivamente, a densidade de presas e predadores.

O sistema (19) no equilíbrio desejadoé dado por

onde

$$
\begin{aligned}
& r_{1}-a_{11} x_{1}^{*}-a_{12} x_{2}^{*}=0, \\
& r_{2}-a_{21} x_{1}^{*}-a_{22} x_{2}^{*}+u^{*}=0,
\end{aligned}
$$

$$
u^{*}=-r_{2}+a_{21} x_{1}^{*}+a_{22} x_{2}^{*} .
$$

Este problema do controle consiste em determinar a função de controle $u$ que transfere $o$ sistema (19) do estado inicial:

ao estado final:

$$
Y(0)=Y_{0},
$$

$$
Y(\infty)=0,
$$

minimizando o funcional:

$$
J=\int_{0}^{\infty}\left[Y^{T} Q Y+r u^{2}\right] d t,
$$

onde $Y=\left[\begin{array}{l}x_{1}-x_{1}^{*} \\ x_{2}-x_{2}^{*}\end{array}\right]$ e $Q \in \mathbb{R}^{2 \times 2}$ é positiva definida e $r$ é uma constante a ser calculada.

De acordo com a programação dinâmica o problema do controle ótimo formulado reduz-se à resolução da equação de Hamilton-JacobiBellman (Molter \&Rafikov, 2004):

$$
\mathcal{H}(x, u)=\min _{u \in \Phi}\left(\frac{d s}{d t}+w\right)
$$




$$
=\left(\frac{d s}{d t}+w\right)_{u=u^{o}}=0,
$$

ondeФé o conjunto das funções de controle admissíveis, $u^{0}$ é o ótimo do controle e

$$
w=Y^{T} Q Y+r u^{2} .
$$

Em Molter, Rafikov (2014) é mostrado que afunção

$$
S\left(x_{1}, x_{2}\right)=\sum_{i=1}^{2} c_{i}\left(x_{i}-x_{i}^{*}-x_{i}^{*} \ln \left(\frac{x_{i}}{x_{i}^{*}}\right)\right)
$$

éuma função de Lyapunov para sistemas populacionais, satisfazendo a condição final:

$$
S(\infty)=0
$$

A derivada total de $S$ em relação a $t$ é dada por:

$$
\begin{gathered}
\frac{d s}{d t}=c_{1}\left(x_{1}-x_{1}^{*}\right)\left(-a_{11}\left(x_{1}-x_{1}^{*}\right)-a_{12}\left(x_{2}-x_{2}^{*}\right)\right)+ \\
\quad c_{2}\left(x_{2}-x_{2}^{*}\right)\left(-a_{21}\left(x_{1}-x_{1}^{*}\right)-a_{22}\left(x_{2}-x_{2}^{*}\right)+u\right) .
\end{gathered}
$$

Substituindo as equações (21) e (22) na equação (20), obtém-se:

$$
\begin{aligned}
& \min _{u \in \Phi}\left\{\left(c _ { 1 } ( x _ { 1 } - x _ { 1 } ^ { * } ) \left(-a_{11}\left(x_{1}-x_{1}^{*}\right)-a_{12}\left(x_{2}-\right.\right.\right.\right. \\
& \left.\left.x_{2}^{*}\right)\right)+c_{2}\left(x_{2}-x_{2}^{*}\right)\left(-a_{21}\left(x_{1}-x_{1}^{*}\right)-a_{22}\left(x_{2}-\right.\right. \\
& \left.\left.\left.\left.x_{2}^{*}\right)+u\right)+Y^{T} Q Y+r u^{2}\right)\right\}=0 .
\end{aligned}
$$

A função $u$ pode ser obtida da seguinte condição:

então,

$$
\frac{\partial[\mathcal{H}(x, u)]}{\partial u}=0,
$$

$$
u=-\frac{c_{2}\left(x_{2}-x_{2}^{*}\right)}{2 r}
$$

Substituindo a equação (24) em (23) temos:

$$
\begin{gathered}
-c_{1} a_{11}\left(x_{1}-x_{1}^{*}\right)^{2}-c_{1} a_{12}\left(x_{1}-x_{1}^{*}\right)\left(x_{2}-x_{2}^{*}\right)- \\
c_{2} a_{21}\left(x_{1}-x_{1}^{*}\right)\left(x_{2}-x_{2}^{*}\right)-c_{2} a_{22}\left(x_{2}-x_{2}^{*}\right)^{2}- \\
\frac{c_{2}^{2}\left(x_{2}-x_{2}^{*}\right)^{2}}{2 r}+\frac{c_{2}^{2}\left(x_{2}-x_{2}^{*}\right)^{2}}{4 r}+q_{11}\left(x_{1}-x_{1}^{*}\right)^{2}+q_{21}\left(x_{1}-\right. \\
\left.x_{1}^{*}\right)\left(x_{2}-x_{2}^{*}\right)+q_{12}\left(x_{1}-x_{1}^{*}\right)\left(x_{2}-x_{2}^{*}\right)+q_{22}\left(x_{2}-\right. \\
\left.x_{2}^{*}\right)^{2}=0 .
\end{gathered}
$$

Comparando os termos similares, obtemos o seguinte sistema:

$$
\begin{aligned}
& -c_{1} a_{11}+q_{11}=0, \\
& -c_{1} a_{12}-c_{2} a_{21}+q_{21}+q_{12}=0, \\
& -c_{2} a_{22}-\frac{c_{2}^{2}}{2 r}+q_{22}+\frac{c_{2}^{2}}{4 r}=0 .
\end{aligned}
$$

Para realizar a simulação numérica foram considerados os seguintes valores: $q_{11}=3,75 \times$ $10^{-5}, q_{12}=q_{21}=0$ e $q_{22}=1,25 \times 10^{-2}$. A partir do sistema (33) foram calculados $c_{1}=0,0417$, $c_{2}=1$ e $r=20$.

As trajetórias temporais do sistema de LotkaVolterra com controle (19) são apresentadas na Figura 9.

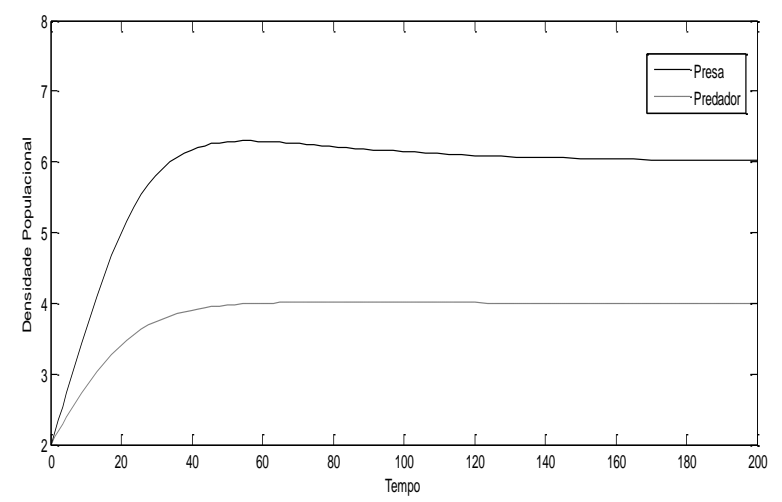

Figura 9: Trajetórias do Sistema de LotkaVolterra com controle.

A Figura 9 mostra que o sistema controlado aproxima-se do seu estado de equilíbrio em torno de 25 dias. Verificou-se em outras simulações que, para este modelo, o tempo que as pragas levam para atingir o nível desejado depende dos parâmetros da matriz $Q$ e o valor de $r$. É possível reduzir o tempo gasto para atingir esse nível, no entanto isto também pode levar a picos de densidades de pragas em momentos anteriores ao equilíbrio, o que não seria desejado por causarem danos à lavoura.

Observe que nesta seção foi realizado o controle não linear somente para o modelo de Lotka-Volterra, visto que para o modelo de Holling-Tanner não é possível realizar esse tipo de controle (Schmid, 2005).

\section{Conclusões e Considerações}

Este trabalho propôs a formulação e resolução de problemas de controle ótimo de sistemas não lineares presa-predador de Lotka-Volterra e de Holling-Tanner. Foram utilizadas três metodologias de controle; o controle linear para 
sistemas linearizados, o controle linear para sistemas não linearizados e o controle não linear.

Os problemas de controle foram aplicados no cultivo do milho, onde se percebeua necessidade do uso de controle, pois os sistemas estavam naturalmente equilibrados numa densidade de pragas bem acima do considerado abaixo de danos econômicos.

Na literatura sobre o cultivo de milho não foram encontradas muitas informações sobre a aplicação da modelagem matemática no controle biológico de pragas. Neste sentido, este trabalho pretende dar sua contribuição. No entanto, devido a esta deficiência, as informações sobre coeficientes dos modelos matemáticos são poucos, e para alguns deles não se encontrou nenhuma informação. Talvez esta tenha sido a maior dificuldade em empregar a metodologia proposta.

Das técnicas de controle utilizadas podem-se destacar algumas particularidades de cada uma. Do controle linear para sistemas linearizados a linearização do sistema pode ser vista como uma desvantagem em relação às demais técnica, pois pode ocorrer o cancelamento de termos importantes da descrição do sistema. No entanto esta é uma técnica bastante difundida pela sua simplicidade no uso de equações puramente lineares. Já do controle linear em sistemas não lineares pode-se observar que é uma técnica promissora para modelos complexos apesar de apresentar desvantagem como a utilização de dados heurísticos em sua implementação, que são as matrizes $Q$ e $R$, uma vez que a eficiência dos resultados depende destas matrizes. Na técnica de controle não linear utilizada percebe-se uma redução na utilização destes dados heurísticos, pois os valores de $r$ e $c$ são calculados. No entanto, é uma técnica limitada em sua implementação, pois não é aplicável para qualquer modelo não linear.

Na forma geral pôde-se observar que os sistemas estabilizaram num período de tempo próximo dos 20 dias. O que mostrou que para os modelos utilizados o controle de pragas aplicado ao cultivo do milho foi eficiente. $\mathrm{O}$ controle estabilizou os sistemas em um nível desejado de densidade de pragas, fazendo com que o sistema ficasse equilibrado biologicamente e abaixo do limiar de danos econômicos a agricultura.

\section{Agradecimentos}

A autora Jéssica C. S. Buenoagradece àUFPel pelo apoio financeiro recebido.

\section{Referências}

BASSANEZI, R. C. Ensino-Aprendizagem com Modelagem Matemática. São Paulo, Editora Contexto, 2002.

BOYCE, W. E.; DIPRIMA, R. C. Equações Diferenciais Elementares e Problemas deValores de Contorno. São Paulo,Editora LTC, 2002.

CAETANO, M. A. L.; YONEYAMA, T. Optimal and sub-optimal control in Dengue epidemics.Optimal Control Applications And Methods, V. 22, p. 63-73, 2001.

CARDOSO, R. T. N.; CRUZ, A. R.;WANNER, E. F.; TAKAHASHI, R. H. C. Multi-Objective Evolutionary Optimization of Biological Pest Control with Impulsive Dynamics in Soybean Crops. Bulletin of Mathematical Biology, V. 71, 6, p. 1463-1481, 2009.

CRUZ, I.; MONTEIRO, M. A. R. Controle Biológico da lagarta do cartucho do milho Spodoptera frugiperda utilizando o parasitoide de ovos Trichogrammapretiosum. Sete Lagoas, dez. 2004. Comunicado técnico 114. Acessadoemjan. 2014. Online. Disponível em

http://www.agencia.cnptia.embrapa.br/recurs os/Comunicado114ID-mk8B5WBhWn.pdf

HOLLING, C. S. The components of predation as revealed by a study of small-mammal predation of the European pine sawfly.Can. Entomol, V. 91,p. 293-320, 1959.

LOTKA, A. J. Elements of physical biology.Baltimore, Williams and Wilkins, 1925.

MOLTER, A.; RAFIKOV, M. Controle Ótimo para um Sistema Caótico de Lotka-Volterra. Tendências em Matemática Aplicada e Computacional, V.5, n. 2, p.237-245, 2004.

MOLTER, A., RAFIKOV, M. Controle ótimo em Agroecosistemas usando SDRE. Tendências em Matemática Aplicada e Computacional TEMA 3, p. 221-232, 2011.
MOLTER,
A.;
RAFIKOV,
M.

Nonlinearoptimalcontrolofpopulation 
systems: applications in ecosystems. Nonlinear Dynamics, V. 76, n. 2, p. 1141-1150, 2014.

NAIDU, D. S. Optimal Control Systems. Idaho, Publisher CRC Press LLC, 2003.

RAFIKOV, M.; BALTHAZAR, J.M.; VON BREMEN, H.F. Mathematical modeling and control of population systems: Applications in biological pest control. In Applied Mathematics and Computation, V. 200, p. 557573, 2008.

RODRIGUES, H. S.;MONTEIRO, M. T. T.; TORRES, D. F. M. Dynamics of Dengue epidemics when using optimal control.Mathematicaland Computer Modelling,V. 52,p. 1667-1673, 2010.

SCHMID, A. B. Controleótimo de sistemaspopulacionaisqueexibemcaos.

Dissertação (Mestrado em Modelagem Matemática) - Curso de Pós-graduação em Modelagem Matemática, Universidade Regional do Noroeste do Estado do Rio Grande do Sul, 2005.

TANG, S.; CHEKE, R.A. Models for integrated pest control and their biological implications.Math.Biosci.,V. 215, p. 115125,2008 .

TANNER, J. T.The stability and the intrinsic growth rates of prey and predator populations.Ecology, V. 56, p. 855-867, 1975.

TUSSET, A.; RAFIKOV, M. Controle Ótimo de Pragas: Modelos Linearizados, Funcional Quadrático. Tendências em Matemática Aplicada e Computacional, V. 5, n. 1, p. 145154, 2004.

PEREIRA, L. G. B. Táticas de Controle da Lagartado-Cartucho do Milho, Spodoptera frugiperda. Minas Gerais, out. 2007. Acessadoemjan. 2014. Online. Disponível em: http://sbrt.ibict.br/dossietecnico/downloadsD T/MTk2

VOLTERRA, V. Variazioni e fluttuazionidel numero d'individui in specieanimaliconviventi. Mem. R. Com. Tolassogr, V.131, p. 31-113, 1927. 